\title{
OPEN Physiological responses of Agriophyllum squarrosum and Setaria viridis to drought and re-watering
}

\begin{abstract}
Juanli Chen ${ }^{1,2 \bowtie}$, Xueyong Zhao ${ }^{2}$, Yuqiang Li' ${ }^{2}$, Yongqing Luo ${ }^{2}$, Yaqiu Zhang ${ }^{3}$, Mei Liu ${ }^{1}$ \& Yan Li ${ }^{1}$
Drought resistance of psammophyte determines survival and growth, but their responses to drought are not well understood. We conducted a pot experiment to study how physiological characteristics respond to drought and rehydration. We found that watering to 60-65\% of field capacity (the control) provided more water than was required by Agriophyllum squarrosum and its leaves became yellow and slightly wilted. The total chlorophyll content and Fm (maximum fluorescence after dark adaptation) in control were lower than in the drought treatment, and both decreased after rehydration. With increasing drought duration and intensity, the relative water content (RWC), chlorophyll content, Fm, and the quantum efficiency of photosystem II (Fv/Fm) of Setaria viridis decreased, but malondialdehyde and membrane permeability increased. During the late drought, the activities of three antioxidant enzymes in A. squarrosum increased to prevent membrane lipid peroxidation; for $S$. viridis, only peroxidase and superoxide dismutase activities increased. After rehydration, RWC of both species increased, but $F v / F m$ of $A$. squarrosum and $F m$ of $S$. viridis did not recover under severe drought. Our research illustrated that $A$. squarrosum is better adapted to arid environment than $S$. viridis, but the high soil moisture content is not conducive to normal growth of $A$. squarrosum.
\end{abstract}

The world's arid and semi-arid regions account for about one-third of the land area, versus nearly half of terrestrial land in China ${ }^{1}$. Water in these areas is unevenly distributed both temporally and spatially, and often does not satisfy plant needs ${ }^{2,3}$. Hence, plants are subjected to repeated cycles of drought and rehydration throughout their life cycle by this highly variable climate ${ }^{4}$. To understand plant survival under these conditions, it is necessary to comprehend the mechanisms that underlie plant physiological responses to drought and re-watering. With the global climate changing and the environment deteriorating, water shortages have become increasingly serious, causing a more intense situation on drought stress ${ }^{5}$. Drought causes water loss from plant cells, which leads to major changes in plant morphology, physiology, and biochemistry, and limits the growth range and living space of many species ${ }^{6,7}$. Many plants have evolved multiple mechanisms and strategies to adapt to water deficits through long-term natural selection and coevolution ${ }^{8,9}$.

Water availability limits vegetation in arid ecosystems by affecting photosynthesis, net productivity, and plant survive, both in natural vegetation communities and after ecological restoration ${ }^{3,10}$. Thus, dryland ecosystems respond rapidly to precipitation changes ${ }^{11}$. Studies have shown that plants osmoregulation can be carried out by accumulating osmotic regulator to maintain cell water balance, and our research about $A$. squarrosum and $S$. viridis is consistent with the results ${ }^{12}$. At the same time, many studies have indicated that plants can produce high levels of reactive oxygen species (ROS) under adversity, which results in increased membrane permeability (due to peroxidation of membrane lipids), protein inactivation, and even plant death ${ }^{1,13}$. The accumulation of ROS in plants also activates a protective system of antioxidant enzymes, with increased activities of antioxidant enzymes such as catalase, superoxide dismutase (SOD) and peroxidase, which scavenge excess ROS to maintain a balance between an active oxygen metabolism and reducing the damage to cell membranes caused by $\operatorname{ROS}^{14,15}$. Therefore, plant antioxidant enzyme defense function plays an important physiological role in plant stress resistance.

The effects of drought are intricate due to the different combinations of environmental characteristics and plant characteristics, including species differences ${ }^{1,16}$. Mafakheri et al. ${ }^{17}$ reported that drought dramatically

\footnotetext{
${ }^{1}$ Ecological Security and Protection Key Laboratory of Sichuan Province, Mianyang Normal University, Mianyang 621000, China. ${ }^{2}$ Naiman Desertification Research Station, Northwest Institute of Eco-Environment and Resources, Chinese Academy of Sciences, Lanzhou 730000, China. ${ }^{3}$ Hanzhong Agricultural Technology Extension Center, Hanzhong 723000, China. ${ }^{\circledR}$ email: juanlic@163.com
} 
decreased the contents of the chlorophyll a, chlorophyll b, and total chlorophyll content of three chickpea (Cicer arietinum) cultivars. It was also reported that drought stress reduced the chlorophyll fluorescence of olive, and that the decreased chlorophyll fluorescence in Brassica rapa resulted directly from a failure to recover from drought after rehydration ${ }^{18}$. However, drought promoted an increase in the chlorophyll content of Periploca sepium $^{5}$. The ratio of variable to maximum quantum yield of PSII $(F \mathrm{v} / F \mathrm{~m})$ in barley and sugar beet decreased under drought stress ${ }^{19,20}$, but the values of $F \mathrm{v} / F \mathrm{~m}$ and $F \mathrm{~m}$ in chickpea remained stable ${ }^{21}$. Water deficits increased malondialdehyde and antioxidant enzyme activities in Populus cathayana ${ }^{22}$, but decreased the relative water content (RWC) in Prunus avium ${ }^{23}$. Membrane permeability in Fargesia denudata was weak owing to the higher activities of SOD and catalase ${ }^{24}$. The seed yield in Glycine max was positively correlated with SOD activity ${ }^{25}$, and there was a significant positive correlation between total antioxidant capacity and catalase activity ${ }^{26}$.

Horqin Sandy Land used to be famous grassland, but it has become the largest sandy land in China due to climate change, overharvesting and overgrazing ${ }^{27}$. During vegetation restoration to control the desertification, the succession that occurs in vegetation communities can be divided into three stages ${ }^{28}$ : (1) Pioneer psammophyte such as A. squarrosum invades a site and colonizes the mobile dunes, the small and flat seeds of this species and the rapid growth of the radicle after germination help the seedlings to rapidly become established in the sand. (2) When the dunes have become semi-fixed, a community dominated by Artemisia halodendron has formed, and the vegetation biomass and cover result primarily from its perennial nature. (3) Stabilization of the land surface and the improved soil quality allow succession to climax species dominated by S. viridis and Leymus chinensis on the fixed dunes. The establishment of $A$. squarrosum plays a role in windbreak and sand-fixation, reduces the mobility of the surface matrix, and creates a stable matrix condition for the invasion of other species. The colonization by $A$. halodendron results in the accumulation of organic matter and the improvement of surface soil fertility. The distribution of $S$. viridis expands and its population increases greatly ${ }^{29}$.

Research on the physiological mechanisms that underlie stress resistance in psammophyte has mainly focused on survival of burial by sand, resistance to abrasion by the wind-sand flow, and endurance of drought stress ${ }^{3,30-32}$. To support preservation of natural vegetation or support ecological restoration, researchers who study psammophyte drought resistance have primarily focused on the short-term regulation of natural conditions or artificial transplanting, but it has been difficult to accurately describe the responses of psammophyte to drought ${ }^{3}$. The dominant species $A$. halodendron has been studied ${ }^{1}$ previously. However, researches on the response of the pioneer and climax species to drought stress are rarely discussed. Therefore, a study of the pioneer species A. squarrosum and the climax species $S$. viridis to drought was explored, and our objective was to reveal their adaptions to an extreme desert environment and to support the restoration of desert vegetation in a degraded sandy land. According to the previous research on the response of other species to stress, we hypothesized: (1) adequate water is favorable to the growth of A. squarrosum and $S$. viridis, but severe drought restrains the growth. (2) adequate water irritates the antioxidant enzyme activity, thus holding lower malondialdehyde levels and guarding plants from damages, but severe drought beyond their adjustable extent results in a remarkable augment of malondialdehyde and severe injury.

\section{Materials and methods}

Study area. The study area is located in the Naiman Banner of Inner Mongolia, near the edges of the Horqin Sandy land ( $42^{\circ} 58^{\prime} \mathrm{N}, 120^{\circ} 43^{\prime} \mathrm{E} ; 360 \mathrm{~m}$ elevation). The region has a continental semi-arid monsoon climate. The annual mean temperature is $6.5^{\circ} \mathrm{C}$ and annual potential evaporation average $1935 \mathrm{~mm}$. This region receives $351.7 \mathrm{~mm}$ of precipitation annually, with $70-80 \%$ of the total in the growing season of June to August. The landscape is dominated by a mosaic of various types of dunes and sandy meadows. The zonal soil is a sandy chestnut soil, but the soil in most areas has degraded into an aeolian sandy soil due to the combination of a warm and dry climate with unsustainable human activities. The field capacity of the sandy soil was $13.0 \%$. The main vegetation types are dune and meadow vegetation and the main shrubs are Caragana microphylla, Salix gordejevii and A. halodendron. The main herbaceous plants are A. squarrosum, S. viridis, Artemisia frigida, Salsola collina, Cleistogenes squarrosa and Artemisia sieversiana ${ }^{12}$. The experimental materials were A. squarrosum and S. viridis, and these species comply with institutional, national and international guidelines. The plant materials used in the study were previously isolated and identified by Halin Zhao in our laboratory. The specimens of $A$. squarrosum (Accession No: LZD0004353) and S. viridis (Accession No: LZD0001836) have been deposited in Northwest institute of Eco-environment and Resources, Chinese Academy of Sciences (http://www.nieer.cas.cn/).

Experimental design and measuring. In August 2018 mature seeds of $S$. viridis were collected from fixed dunes. In October of the same year, A. squarrosum seeds were gathered. We sowed the seeds in plastic pots (28 cm in diameter and $21 \mathrm{~cm}$ in height) filled with $8.5 \mathrm{~kg}$ of air-dried and sieved $(0.6 \mathrm{~mm})$ dune soil at the end of April 2019. The young seedlings were thinned after 20 days of germination. Five seedlings of A. squarrosum and ten seedlings of $S$. viridis were retained in each pot after thinning. The remaining seedlings were healthy and similar in size. These plants were cultured under the same conditions and were irrigated with well water until the experiment began. The experiment was conducted at the Naiman Desertification Research Station, Chinese Academy of Sciences under a rain shelter to let us control soil water levels, but otherwise the plants grew under natural conditions. The shelter decreased the light intensity by about $10 \%$ below ambient levels, but was established at a height of $4 \mathrm{~m}$ so that the ambient temperature inside and outside the shelter differed by less than $1^{\circ} \mathrm{C}$.

In mid-July 2019, one hundred and twenty pots of each species were randomly separated into three water treatments: plants were watered to $60-65 \%$ of field capacity (the control), $40-45 \%$ of field capacity (moderate drought, MD), 20-25\% (severe drought, SD) of field capacity. The severity was defined based on the physiological responses of $A$. halondendron to soil water in the previous study ${ }^{1}$. From 17 to 20 July, we regulated soil water content within the specified range based on the weight of soil in each pot. Subsequently, we maintained the water 
content within the range for 11 days (from 21 to 31 July) according to the field capacity (weight basis). On 1, 7 and 13 August (every 6 days after the end of water control), 60 pots were chosen ( 20 replicates each treatment selected randomly) to test the impacts of the sustained drought stress on the physiological indices. The remained 60 pots were well re-watered on 7 and 13 August to determine the effects of re-watering after sustained drought, and excess water flowed out of the hole at the bottom of the pot. After a day of rehydration (on 8 and 14 August), leaves were selected randomly to measure physiological responses. The experiment was repeated for two years and the results showed similar trends.

Analytical methods and statistical analysis. On 1, 7, and 13 days after the initiation of drought treatments, we measured the physiological parameters of the fully developed leaves from the top of the plants selected from the first 60 pots. At the same time, the leaves of the same part as above from the rest 60 pots were chosen on the first day after rehydration on 8 and 14 August. The chlorophyll fluorescence (described below) was detected on the sampling date, and then the leaves were sampled to assay the degree of peroxidation of membrane-lipids and the activity levels of key antioxidant enzymes.

Chlorophyll was extracted from the leaves using $95 \%$ ethanol, and its content was determined at absorbance values of 665,649 and $470 \mathrm{~nm}^{33}$. The chlorophyll fluorescence of dark and light adaptive leaves was stimulated, and determined with a portable chlorophyll fluorometer. (Hansatech, England) ${ }^{34}$. The minimum fluorescence with all photosystem II (PSII) centers open $(F o$ ) was measured under the condition of dark adaption for 30 min, and the maximum dark-adapted fluorescence $(\mathrm{Fm})$ was measured by saturation pulse irradiation at $2800 \mu \mathrm{mol} /$ $\left(\mathrm{m}^{2} \mathrm{~s}\right)$. Variable fluorescence $(F \mathrm{v})$ equaled the difference between $F \mathrm{~m}$ and $F_{\mathrm{O}}$. The maximum efficiency of PSII equaled $\mathrm{Fv} / \mathrm{Fm}^{35}$.

RWC $=[($ Fresh Weight - Dry Weight $) /($ Turgid Weight - Dry Weight $)] \times 100 \%$. RWC was used to reflect leaf water status ${ }^{36}$.

Random $1 \mathrm{~g}$ leaves were extracted with buffer solutions and centrifugation, and the supernatant was stored at $4{ }^{\circ} \mathrm{C}$ for assaying malondialdehyde content and the activities of catalase, SOD, and peroxidase ${ }^{1}$. Catalase activity was expressed as the quantity of enzyme needed to eliminate $10^{-6} \mathrm{~mol}_{2} \mathrm{O}_{2}$ in $1 \mathrm{~min}^{37}$. SOD activity was expressed as the quantity of enzyme needed to prevent the reducing of nitroblue tetrazolium ${ }^{38}$. Peroxidase activity was defined as the increase of the oxidation of guaiacol absorption of 0.01 one $\mathrm{min}^{3}$. The malondialdehyde content was quantified using the method of Heath et al. ${ }^{39}$. We have determined the above colorimetric analysis with UV-1601 UV-Visible Spectrophotometer (Shimadzu Corporation, Japan).

All data analyses were performed with the SPSS software (version 20.0) (http://www.ibm.com/software/analy tics/spss/) and the graphs were drawn using version 12.5 of SigmaPlot (https://systatsoftware.com/). One-way analysis of variance (ANOVA) was performed to compare differences among the treatments. We used Fisher'sleast-significant-difference (LSD) test to detect significant differences between pairs of drought levels and used Pearson's correlation coefficient (r) to identify relationships among physiological features.

\section{Results}

Changes in chlorophyll content and fluorescence. The total chlorophyll content of A. squarrosum in the control decreased gradually with increasing drought duration (Fig. 1A1), but the contents under moderate and severe drought first increased (from 1 to 7 August) and then decreased (from 7 to 13 August). During the same periods, the total chlorophyll content under moderate drought was significantly higher than that in the control, but significantly less than that under severe drought. The total chlorophyll content of S. viridis (Fig. 1A2) decreased with increasing drought duration and intensity, and the reduction from 1 to 7 August was greater than that from 7 to 13 August. After re-watering on 8 and 14 August, the total chlorophyll contents of both studied species were significantly lower than those before rehydration. In addition, the total chlorophyll content of $A$. squarrosum was in the order of severe drought $>$ moderate drought $>$ Control, and the order of $S$. viridis was severe drought $<$ moderate drought $<$ Control.

Fm of A. squarrosum (Fig. 1B1) first increased (from 1 to 7 August) and then decreased (from 7 to 13 August) in all treatment. On 7 August, Fm under severe drought reached the highest value (1309.1). In contrast, Fm of $S$. viridis (Fig. 1B2) decreased as drought intensity and duration increased, and the reduction from 7 to 13 August was lower than that from 1 to 7 August. On 8 and 14 August, rehydration decreased the Fm of A. squarrosum, and Fm on 14 August was significantly lower than that on 8 August. On 14 August, Fm was lowest in the control (735.4), which was $20.3 \%$ and $27.4 \%$ lower than those under moderate and severe drought, respectively. On 8 August, Fm of $S$. viridis was higher than that before rehydration, but the opposite was true on 14 August.

With increasing drought duration and intensity, Fv/Fm of both species (Fig. 1C1, C2) slowly decreased (from 1 to 7 August) and then rapidly decreased (from 7 to 13 August). On 13 August, Fv/Fm was lowest under severe drought, and was significantly lower than in the control and under moderate drought. On 1 August, there was no significant difference in $F \mathrm{v} / F \mathrm{~m}$ of $A$. squarrosum among the treatments, but $F \mathrm{v} / F \mathrm{~m}$ of $S$. viridis was significantly higher in the control and under moderate drought than under severe drought. In addition to Fv/Fm of A. squarrosum in the control and under moderate drought on 14 August, rehydration led to a remarkable increase in $F \mathrm{v} / \mathrm{Fm}$ of both species on 8 and 14 August. $F v / F m$ of $A$. squarrosum was in the sequence of severe drought $>$ moderate drought $>$ Control, and the sequence of $S$. viridis was severe drought $<$ moderate drought $<$ Control.

Changes in RWC, malondialdehyde content and membrane permeability. RWC of both species (Fig. 2A1, A2) decreased markedly from 1 to 13 August. RWC of A. squarrosum under moderate drought was significantly higher than those in the control and under severe drought. The RWC of $S$. viridis also decreased with increasing drought intensity. After re-watering on 8 and 14 August, RWC of both species were significantly higher than the corresponding values before rehydration and RWC on 8 August was observably higher than that 

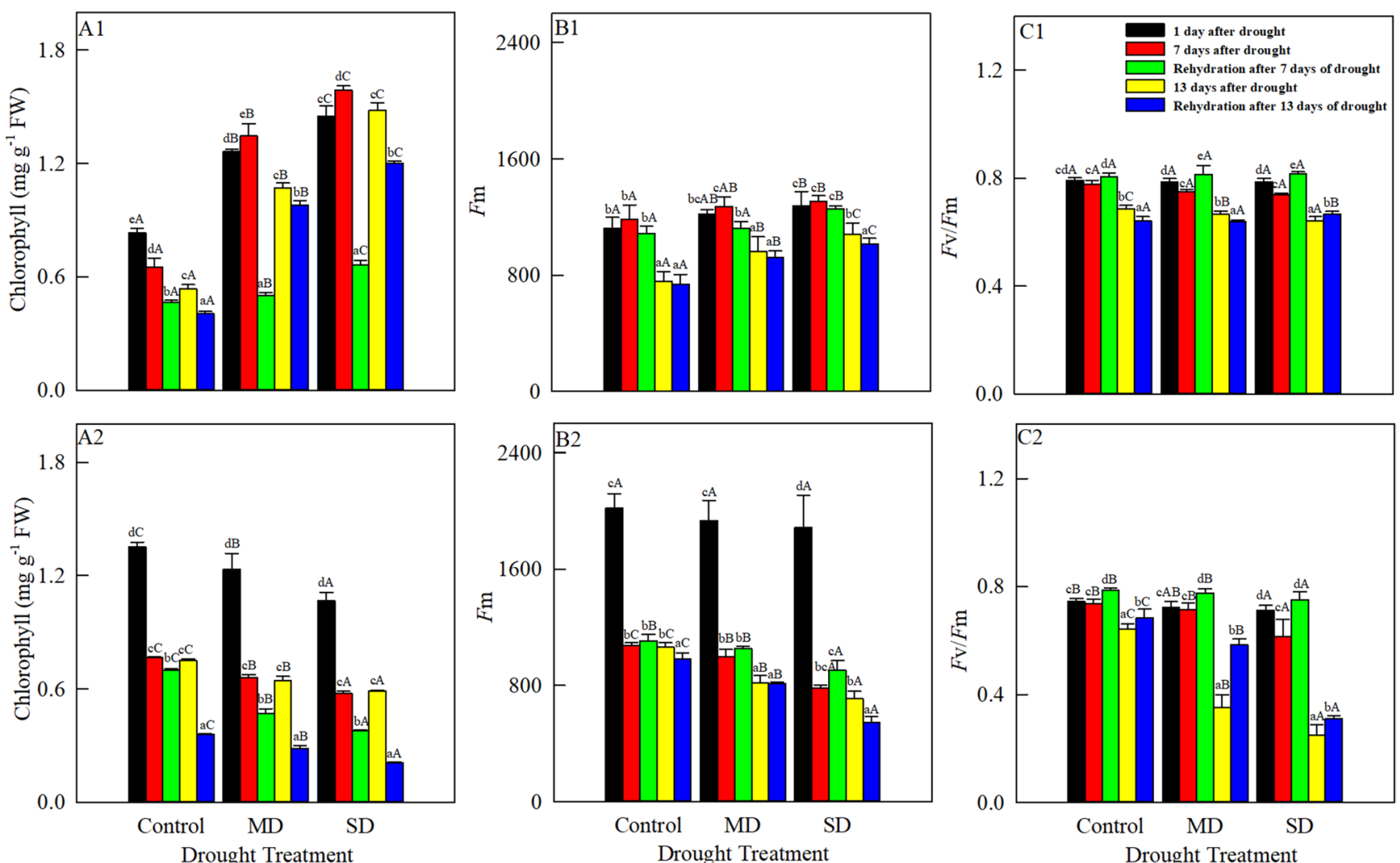

Figure 1. Changes in chlorophyll content, $F \mathrm{~m}$ and $F \mathrm{v} / F \mathrm{~m}$ of $A$. squarrosum (A1, B1, C1) and S. viridis (A2, B2, C2) under drought stress (August 1,7,13) and rehydration (August 8, 14). Different lowercase letters indicate significant differences among days for the same treatment. Different capital letters indicate significant differences among drought treatments for the same day.

on 14 August. RWC of A. squarrosum under severe drought was highest (88.2\%) on 8 August, but RWC of $S$. viridis reached its highest value in the control (93.1\%).

With increasing drought duration, the malondialdehyde content in the control of A. squarrosum (Fig. 2B1) first decreased (from 1 to 7 August) and then increased (from 7 to 13 August), but the content under moderate and severe drought increased continuously. The content decreased with increasing drought intensity on 1 August. However, the contents under moderate drought on 7 and 13 August were significantly higher than those in the control and under severe drought. The malondialdehyde content of $S$. viridis (Fig. 2B2) increased with increasing drought duration and stress, and reached the highest level under severe drought on 13 August (2.714 mmol g-1 DW). Although the malondialdehyde content in the control of A. squarrosum increased after rehydration on 8 August, the contents in both species on 8 and 14 August were lower than those before rehydration.

Membrane permeability of the two species (Fig. 2C1, C2) increased with increasing drought duration and stress. On 1 August, membrane permeability of A. squarrosum (28.2\%) and S. viridis (22.2\%) reached its lowest values in the control, and these values were significantly lower than those under moderate and severe drought. After re-watering on 14 August, rehydration reduced membrane permeability of A. squarrosum in the control, but permeability reached its highest level (63.3\%) on 8 August, and this was significantly lower than the value before rehydration. On 8 and 14 August, membrane permeability of $A$. squarrosum under moderate and severe drought and of $S$. viridis under all treatments was lower than that before rehydration.

Changes in the activity of antioxidase. With increasing drought duration, peroxidase activities of both species (Fig. 3A1, A2) first decreased and then increased, except that the activity in A. squarrosum under severe drought increased continuously. Peroxidase activity of $S$. viridis was the largest $\left(136.07310^{3} \mathrm{U} \mathrm{g}^{-1} \mathrm{DW} \mathrm{min}{ }^{-1}\right)$ in the control on 1 August, 1.15 times and 1.50 times, respectively, the values under moderate and severe drought. After re-watering on 8 and 14 August, peroxidase activity of A. squarrosum was higher than that before rehydration, whereas $S$. viridis showed decreases after both re-hydrations.

SOD activities of both species (Fig. 3B1, B2) increased with increasing drought duration and severity. From 1 to 13 August, their activities were significantly higher under severe drought than in the control and under moderate drought. On 13 August, SOD activity of S. viridis was highest under severe drought, at $173.07110^{3} \mathrm{U} \mathrm{g}^{-1} \mathrm{DW} \mathrm{h}^{-1}$, which was 1.13 and 1.38 times the value in the control and under moderate drought, respectively. SOD activity of $A$. squarrosum after rehydration was higher than that before rehydration, and its activity increased with increasing drought stress. On 8 August, rehydration increased SOD activity in S. viridis, but its activity decreased below that before rehydration on 14 August. 

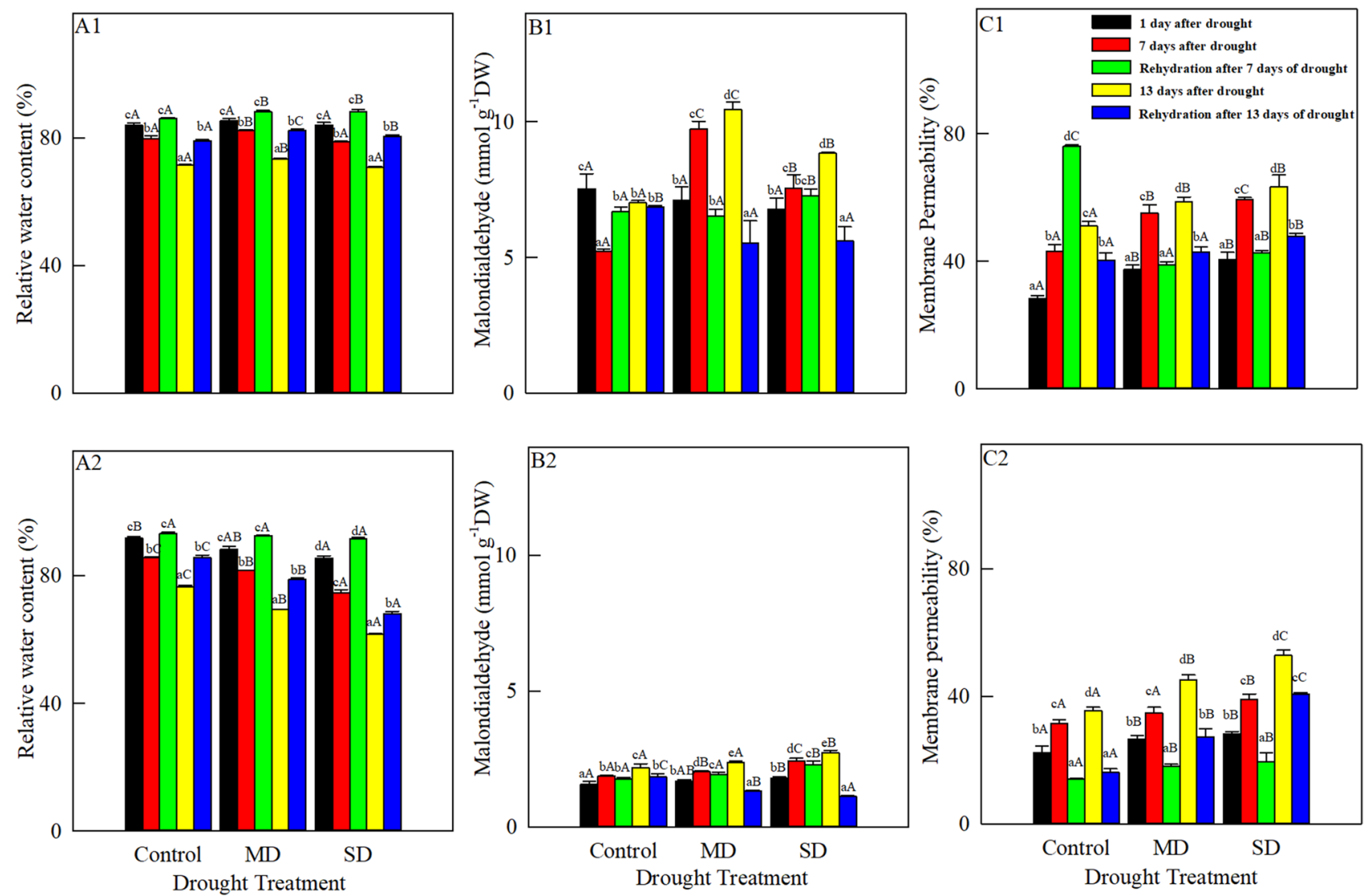

Figure 2. Changes in RWC, malondialdehyde and membrane permeability of A. squarrosum (A1, B1, C1) and S. viridis (A2, B2, C2) under drought stress (August 1, 7, 13) and rehydration (August 8, 14). Different lowercase letters indicate significant differences among days for the same treatment. Different capital letters indicate significant differences among drought treatments for the same day.

With increasing drought duration, catalase activity of the both species (Fig. 3C1, C2) first increased (from 1 to 7 August) and then decreased (from 7 to 13 August), but the catalase activity of A. squarrosum in the control showed the opposite pattern. On 1 August, catalase activity in the control of A. squarrosum was significantly higher than that under moderate drought, but was markedly and significantly lower than that under severe drought. On 7 August, its activity was highest under severe drought $\left(177.416 \mathrm{U} \mathrm{g}^{-1} \mathrm{DW} \mathrm{min}{ }^{-1}\right)$. Catalase activity of $S$. viridis was low, at less than $50 \mathrm{U} \mathrm{g}^{-1} \mathrm{DW} \mathrm{min}{ }^{-1}$ throughout the study period. On 8 and 14 August, rehydration caused an increase in catalase activity of the two plants, except that the activity in the control of $S$. viridis reached its highest level $\left(46.425 \mathrm{U} \mathrm{g}^{-1} \mathrm{DW} \mathrm{min}^{-1}\right)$ on 8 August.

Correlation analysis. Many significant correlations were found among the physiological responses for both species (Table 1). For A. squarrosum, chlorophyll content was significantly positively correlated with Fm and catalase, and catalase was significantly positively correlated with SOD, but peroxidase was significantly negatively correlated with $\mathrm{Fv} / \mathrm{Fm}$ and $\mathrm{Fm}$. Fv/Fm was significantly positively correlated with $\mathrm{Fm}$ and RWC, but negatively correlated with SOD. For $S$. viridis, Fm and peroxidase were significantly positively correlated with chlorophyll content and $\mathrm{Fv} / \mathrm{Fm}$, and RWC was significantly positively correlated with $\mathrm{Fv} / \mathrm{Fm}$ and $\mathrm{Fm}$. Catalase was significantly positively correlated with chlorophyll content and $F \mathrm{v} / F \mathrm{~m}$, but Membrane permeability was negatively correlated with RWC and $F \mathrm{v} / \mathrm{Fm}$. SOD was significantly positively correlated with malondialdehyde, but negatively correlated with peroxidase, total chlorophyll content, $\mathrm{Fv} / \mathrm{Fm}$, and $\mathrm{Fm}$.

\section{Discussion}

Chlorophyll plays an important part in the assimilation, transfer and conversion of light energy during photosynthesis. Its content is therefore closely related to the carbon fixation efficiency of photosynthesis and, because photosynthesis provides the energy source for metabolic responses, plays an important role in the drought resistance of plants. Chlorophyll fluorescence is often used to analyze photosynthesis efficiency under stress ${ }^{1}$. $\mathrm{Fm}$ is the fluorescence output when the reaction center of PSII is completely closed, and therefore reflects the maximum electron transfer through PSII ${ }^{40} . \mathrm{Fv} / \mathrm{Fm}$ represents the energy conversion efficiency of PSII reactions, and can be used to measure the degree of external stress ${ }^{41}$.

The chlorophyll content and Fm of A. squarrosum first increased and then decreased under moderate and severe drought, indicating that $A$. squarrosum adjusted its energy capture during the early stage of drought, and 

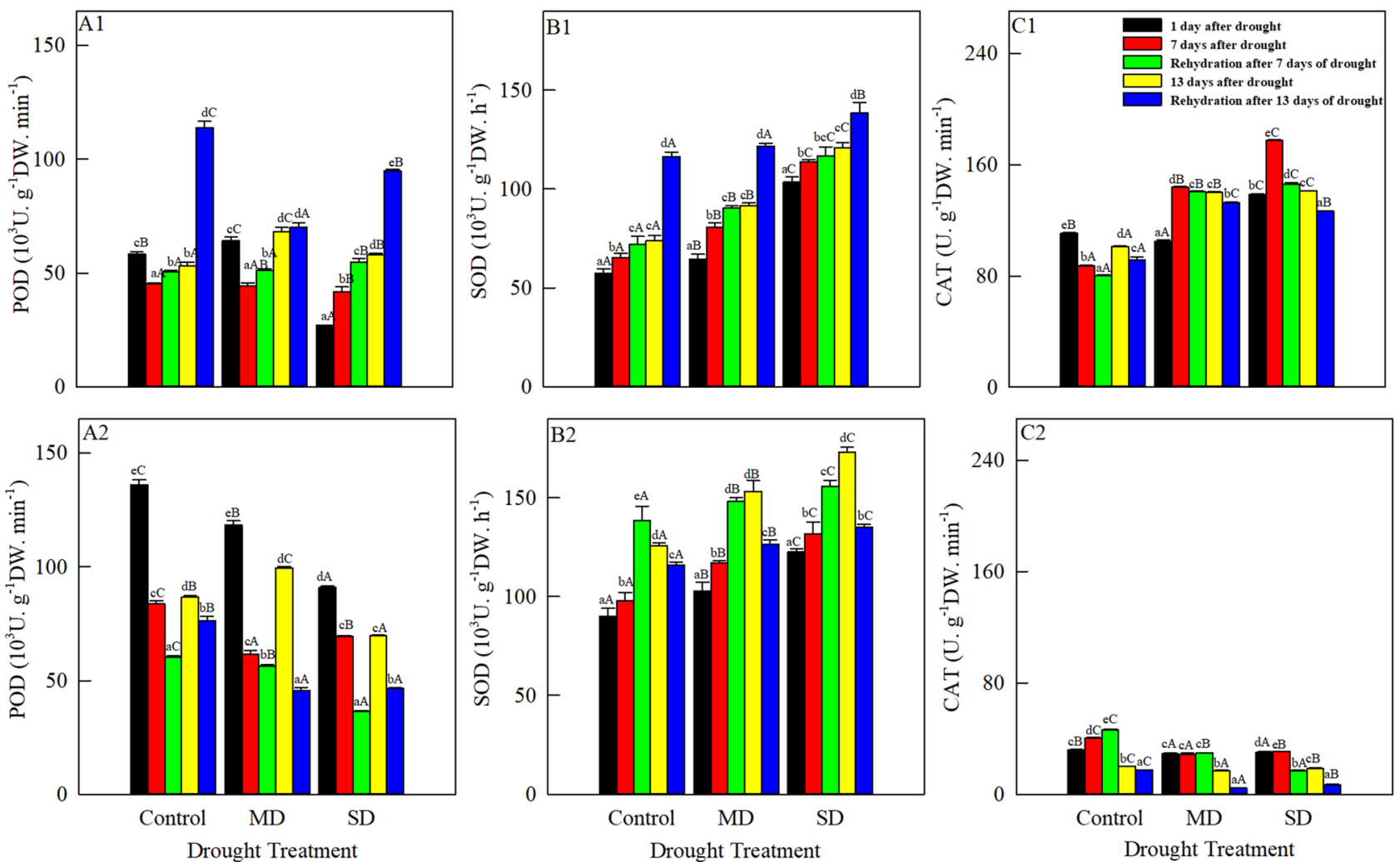

Drought Treatment

Figure 3. Changes in POD, SOD and CAT of A. squarrosum (A1, B1, C1) and $S$. viridis (A2, B2, C2) under drought stress (August 1, 7, 13) and rehydration (August 8,14). Different lowercase letters indicate significant differences among days for the same treatment. Different capital letters indicate significant differences among drought treatments for the same day.

\begin{tabular}{|c|c|c|c|c|c|c|c|c|c|}
\hline Species & Item & Total chlorophyll & $F \mathrm{~m}$ & $F v / F m$ & RWC & MDA & MP & POD & SOD \\
\hline \multirow{8}{*}{ A. squarrosum } & $F \mathrm{~m}$ & $0.520^{*}$ & & & & & & & \\
\hline & $F \mathrm{v} / F \mathrm{~m}$ & -0.167 & $0.700^{* *}$ & & & & & & \\
\hline & RWC & -0.226 & 0.497 & $0.749^{* *}$ & & & & & \\
\hline & MDA & 0.370 & 0.116 & -0.139 & -0.376 & & & & \\
\hline & MP & 0.155 & -0.006 & -0.197 & -0.375 & 0.360 & & & \\
\hline & POD & -0.292 & $-0.685^{\star *}$ & $-0.618^{*}$ & -0.166 & -0.135 & -0.166 & & \\
\hline & SOD & 0.296 & -0.148 & $-0.552^{*}$ & -0.154 & -0.102 & 0.121 & 0.410 & \\
\hline & CAT & $0.657^{* *}$ & 0.461 & -0.054 & -0.039 & 0.400 & 0.076 & -0.301 & $0.514^{*}$ \\
\hline \multirow{8}{*}{ S. viridis } & $F \mathrm{~m}$ & $0.908^{* *}$ & & & & & & & \\
\hline & $F \mathrm{v} / F \mathrm{~m}$ & 0.366 & $0.549^{*}$ & & & & & & \\
\hline & RWC & 0.348 & $0.582^{*}$ & $0.936^{* *}$ & & & & & \\
\hline & MDA & -0.012 & -0.257 & -0.196 & -0.315 & & & & \\
\hline & MP & -0.097 & -0.403 & $-0.835^{* *}$ & $-0.930^{* *}$ & 0.424 & & & \\
\hline & POD & $0.879^{* *}$ & $0.761^{* *}$ & 0.124 & 0.130 & -0.006 & 0.043 & & \\
\hline & SOD & $-0.569^{*}$ & $-0.633^{\star}$ & $-0.537^{*}$ & -0.456 & $0.542^{\star}$ & 0.350 & $-0.575^{\star}$ & \\
\hline & CAT & $0.576^{*}$ & 0.465 & $0.610^{*}$ & $0.550^{*}$ & 0.138 & -0.341 & 0.344 & -0.343 \\
\hline
\end{tabular}

Table 1. Values of Pearson's correlation coefficient $(r)$ for the relationships among the physiological characteristics for all drought durations, including values after rehydration, in A. squarrosum and $S$. viridis. Abbreviations: $F_{\mathrm{m}}$, maximum chlorophyll fluorescence; $F_{\mathrm{v}}$, variable chlorophyll fluorescence; $F_{\mathrm{v}} / F_{\mathrm{m}}$, quantum efficiency of photosystem II; RWC, relative water content; MDA, malondialdehyde; MP, membrane permeability; POD, peroxidase; SOD, superoxide dismutase; CAT, catalase. ${ }^{*}$ and ${ }^{\star *}$ indicate a significant correlation at $P<0.05$ and $P<0.01$, respectively. 
because electron transfer was relatively stable, normal photosynthesis was maintained. As stress intensified during prolonged drought, chlorophyll degradation accelerated and electron transfer through PSII slowed, which was similar to the effect of drought stress on chlorophyll of $A$. halodendron ${ }^{1}$. On 1 August, when the drought treatments began, the leaves of A. squarrosum in the control became noticeably yellow and slightly wilted, and the chlorophyll content and Fm were lower than those in the drought treatments. After re-watering, the chlorophyll content and Fm of A. squarrosum decreased, but they increased with increasing drought intensity. There is a limit to plant demand for water, and both too much and too little water are not conducive to plant growth. As a pioneer species during vegetation succession in sandy land, A. squarrosum is a xerophyte ${ }^{31}$. The soil moisture content in the control was higher than its requirements, and its photosynthesis was obviously adversely affected by controlling the water content at a higher level than the plants required. There was a significant positive correlation between $\mathrm{Fv} / \mathrm{Fm}$ and RWC of the two plants, which indicated that water deficit was the main reason for the decrease of $F \mathrm{v} / F \mathrm{~m}$. The chlorophyll fluorescence of $A$. squarrosum could maintain higher photosynthetic performance under drought stress because of its stronger water holding capacity than that of $S$. viridis. For $A$. squarrosum, $\mathrm{Fv} / \mathrm{Fm}$ decreased with increasing drought duration and intensity. This is because drought reduced the electron transfer capacity of PSII and photochemical activity, leading to excessive accumulation of excitation energy, and adversely affecting photosynthesis. $\mathrm{Fv} / \mathrm{Fm}$ increased after re-watering on 8 August, when the reduction of stress slowed the inhibition of photosynthesis by drought, by decreasing the inhibition of photosynthesis.

For $S$. viridis, the chlorophyll content, $F \mathrm{~m}$ and $\mathrm{Fv} / \mathrm{Fm}$ decreased with increasing drought duration and intensity, indicating that drought stress hindered the biosynthesis of chlorophyll, and that chlorophyll decomposition increased, leading to decreased chlorophyll content. At the same time, drought resulted in the decrease of PSII photochemical transformation efficiency and photosynthetic activity, and the damage of PSII receptor, which contributed to the damage of photosynthesis and the decrease of electron transfer ability. Fm and Fv/Fm of $S$. viridis increased after re-watering on 8 August, showing that rehydration relieved the drought stress. In addition, $\mathrm{Fv} / \mathrm{Fm}$ increased and Fm decreased after re-watering on 14 August, suggesting that the damage to PSII was mitigated by rehydration, but the electron transfer in the PSII reaction center continued to be slower than normal. The chlorophyll content of $S$. viridis did not return to normal after re-watering, indicating that the leaves of $S$. viridis were damaged by both prolonged and severe drought stress and that chlorophyll synthesis was significantly affected ${ }^{1}$.

The cell membrane is both a dynamic barrier between the cell interior and its surroundings, and a channel for the exchange of substance and information with its environment ${ }^{42}$. In particular, it controls water transport between the cell and its environment, leading to changes in RWC. RWC can be used to indicate the degree of dehydration of cells and assess the level of drought suffered by plants ${ }^{43}$. Under drought stress, the loss of water in plants is directly related to the stability of the cell membrane, and a stable cell membrane is the most basic requirement for maintaining sufficient water to support the cell's physiological functions. ROS are produced in large quantities under stress, and this can trigger or exacerbate peroxidation of membrane lipids to produce malondialdehyde. Malondialdehyde can damage the membrane and functional molecules such as proteins and nucleic acids in cells, leading to damage or destruction of the membrane's structure and functions. This, in turn, can increase the permeability of the membrane, leading to growth inhibition or even death. Therefore, changes in membrane permeability and the malondialdehyde content can reflect the degree of membrane lipid peroxidation and cell damage under stress ${ }^{1,3,32}$. It is consistent with our correlation analysis that RWC of the two species is negatively correlated with malondialdehyde and membrane permeability, and the correlation between RWC and membrane permeability in S. viridis is significant.

In A. squarrosum, membrane permeability in the control on 1 August was significantly less than those under moderate and severe drought, but the malondialdehyde content did not differ among the treatments. The change of membrane permeability may have resulted from degreasing of membrane lipids and destruction of the membrane structure after phospholipid dissociation ${ }^{44}$. From 1 to 13 August, malondialdehyde content of $A$. squarrosum in the control first decreased and then increased, while membrane permeability increased continuously, indicating that membrane lipid peroxidation was significantly alleviated in wet soil after short-term drought. In contrast, the severe water deficit during the late stage of drought increased peroxidation of membrane lipids and malondialdehyde accumulation, suggesting that the cell membranes in the control had been damaged during the drought process. The malondialdehyde content and membrane permeability of A. squarrosum increased in the control after rehydration on 8 August, but decreased after rehydration on 14 August. This suggests that rehydration during the early stages of drought can exacerbate the peroxidation of membrane lipids and damage the cell membrane, but that rehydration during the late stages of drought mitigated the stress and eased the damage. Many studies showed that membrane permeability and the malondialdehyde content increased synchronously under stress ${ }^{1}$, but this contradicts our results for A. squarrosum in the control. This may be because the high soil moisture content in the control was not conducive to normal growth of this xerophyte. That is, long-term natural selection in the species' arid sandy environment would lead to continuous adaptation to its environment, allowing A. squarrosum to become widely distributed in the mobile dunes of the Horqin sandy land ${ }^{45}$. With increasing drought duration, the malondialdehyde content and membrane permeability of A. squarrosum increased under both moderate and severe drought, indicating that the accumulation of malondialdehyde after drought stress damaged cell membrane and increased its permeability. The RWC values of $A$. squarrosum in the control were similar, but the membrane permeability fluctuated greatly. This can be due to more than adequate amount of irrigation.

Setaria viridis is a late-successional species, and showed different responses to drought. With increasing drought duration and intensity, RWC of S. viridis decreased, while MDA and membrane permeability increased simultaneously. The results indicated that the early occurrence of water stress and membrane peroxidation in S. viridis under stress was one of the main physiological reasons for its inferior drought tolerance to A. squarrosum. Moreover, the damage degree of plants under drought stress should take into account not only the change 
of membrane permeability, but also the degree of membrane peroxidation and the ability of plant cell membrane to tolerate membrane lipid peroxidation. The chlorophyll content, Fm and Fv/Fm of $S$. viridis decreased with increasing drought duration and severity, and $F \mathrm{v} / F \mathrm{~m}$ of $S$. viridis was significantly negatively correlated with membrane permeability, which increased with increasing drought stress. This indicated that membrane lipid peroxidation and the accumulation of ROS under drought stress damaged the membrane and inhibited photosynthesis. Re-hydration of $S$. viridis increased RWC on both dates and in all drought treatments. This was accompanied by decreased malondialdehyde content, particularly after the 14 August re-watering, and by decreased membrane permeability. Rehydration reduced membrane lipid peroxidation, but it did not return to the control level, showing that drought caused a certain degree of damage that may be permanent or that may take some time to be repaired ${ }^{3}$.

Stress can disrupt the balance of ROS metabolism in aerobic plants. When the concentrations of ROS are too high, peroxidation of membrane lipids and the equilibrium for exchanges of cell materials is also disrupted, resulting in a series of physiological and metabolic disorders. To counteract these disorders, plants have evolved protective enzymes during long-term evolution. The enzymes can eliminate $\mathrm{O}^{2-}, \mathrm{H}_{2} \mathrm{O}_{2}, \mathrm{OH}^{-}$and $\mathrm{O}^{-}$and reduce the damage they cause to the plant ${ }^{46}$. The changes in antioxidant enzyme activities of both species differed under drought stress. SOD played an active role during initial protection against membrane lipid peroxidation and its activity in A. squarrosum increased gradually during the drought. Under natural drought condition, SOD activities of the two species increased gradually, indicating that SOD activity was easily induced by drought stress. At the end of natural drought, the three enzymes of A. squarrosum maintained high level, and the combination of enzymes could resist drought stress, while only POD and SOD in S. viridis were enhanced to alleviate membrane lipid peroxidation. This transformation of the coordination of enzyme activity may be an important physiological mechanism of drought tolerance of A. squarrosum was stronger than that of $S$. viridis under severe drought. On 7 August, the peroxidase and catalase activities decreased in the control. Because ROS are a metabolism by-product of photorespiration, photosynthesis was inhibited by short-term drought, and the decreased accumulation of ROS caused by protective antioxidant enzymes reduced membrane lipid peroxidation by decreasing levels of malondialdehyde ${ }^{47}$. On 7 and 13 August, the activities of protective enzymes in A. squarrosum under moderate and severe drought were greater than that in the control. Drought stress led to the accumulation of ROS, and increased membrane lipid peroxidation, as reflected by the malondialdehyde content. At the same time, the accumulated ROS also stimulated the antioxidant enzyme protection system to continuously increase the activities of enzymes, so as to maintain balance of $\operatorname{ROS}^{48}$.

Setaria viridis showed different responses. From 1 to 13 August, its peroxidase activity first decreased and then increased, but catalase activity showed the opposite pattern, and SOD activity increased gradually, indicating the existences of coordination among these enzymes under drought stress ${ }^{49}$. When catalase activity weakened, SOD and peroxidase activities compensated for this weakness to scavenge more ROS and mitigate cell membrane damage. The catalase activity in $S$. viridis remained less than $50 \mathrm{U} \mathrm{g}^{-1} \mathrm{DW} \mathrm{min}{ }^{-1}$ throughout the study. After rehydration, catalase activity in the control was significantly greater than those under moderate and severe drought. There was a close relationship between $F \mathrm{v} / \mathrm{Fm}$ and catalase activity in $S$. viridis. It is possible that the enzyme must be contributing through ROS scavenging. Some of the antioxidant enzymes of both species did not recover after rehydration, which may be related to the possibility that in xerophytes, rehydration did not immediately improve physiological metabolism. It is possible that their antioxidant enzyme systems were so damaged that they would take longer than our study period to return to normal levels, and our samples were collected 1 day after rehydration.

\section{Conclusions}

Hypothesis 1 and 2 were both partially demonstrated. Adequate water was not conducive to the growth and had negative effects on chlorophyll fluorescence and antioxidant enzymes. Severe drought had negative impact on these indices. Through the above analysis and discussion of the research, we concluded that A. squarrosum is better adapted to arid environment than S. viridis, which is why A. squarrosum can survive on mobile dunes, whereas $S$. viridis can only be colonizes when dunes become fixed dunes. With increasing drought intensity, its chlorophyll content and Fm increased, whereas S. viridis showed the opposite trend. In addition to the control of A. squarrosum, malondialdehyde content and membrane permeability of both species enhanced synchronously with increasing drought duration, demonstrating adverse effects of drought stress. The activities of peroxidase, SOD and catalase in A. squarrosum collectively reduced membrane lipid peroxidation under drought stress, whereas $S$. viridis mainly alleviated membrane damage through peroxidase and SOD. Drought stress caused different degrees of damage to the two plants, and both failed to fully recover after rehydration during the study period, although full recovery might have occurred over a longer time. In future research, it will be necessary to clarify the stress resistance mechanisms of A. squarrosum and S. viridis by examining physiological responses such as stomatal transpiration and cellular processes such as adjustments in cell osmotic potential, turgor pressure, and compatible solutes.

Received: 29 January 2021; Accepted: 30 August 2021

Published online: 20 September 2021

\section{References}

1. Chen, J. L. et al. Effects of drought and rehydration on the physiological responses of Artemisia halodendron. Water 11, 793 (2019).

2. Huxman, T. E. et al. Precipitation pulses and carbon fluxes in semiarid and arid ecosystems. Oecologia 141, 254-268 (2004).

3. Luo, Y. Y. et al. Physiological acclimation of two psammophytes to repeated soil drought and rewatering. Acta Physiol. Plant. 33, 79-91 (2011). 
4. Miyashita, K. \& Kimura, K. Recovery responses of photosynthesis, transpiration, and stomatal conductance in kidney bean following drought stress. Environ. Exp. Bot. 53, 205-214 (2005).

5. An, Y. Y. et al. Organ-dependent responses of Periploca sepium to repeated dehydration and rehydration. S. Afr. J. Bot. 77, 446-454 (2011).

6. Ditmarová, L. et al. Physiological responses of Norway spruce (Piceaabies) seedlings to drought stress. Tree Physiol. 30, 205-213 (2010).

7. Moore, J. P. et al. Adaptations of higher plant cell walls to water loss: Drought vs desiccation. Physiol. Plant. 134, 237-245 (2010).

8. Bacelar, E. et al. Immediate responses and adaptive strategies of three olive cultivars under contrasting water availability regimes: Changes on structure and chemical composition of foliage and oxidative damage. Plant Sci. 170, 596-605 (2006).

9. Seki, M. et al. Regulatory metabolic networks in drought stress responses. Curr. Opin. Plant Biol. 10, 296-302 (2007).

10. Weltzin, J. F. \& Mcpherson, G. R. Implications of precipitation redistribution for shifts in temperate savanna ecotones. Ecology 81, 1902-1913 (2000).

11. Xu, H., Li, Y. G. \& Zou, T. Ecophysiological response and morphological adjustment of two Central Asian desert shrubs towards variation in summer precipitation. Plant Cell Environ. 30, 399-409 (2010).

12. Chen, J. L. et al. Responses to drought stress in germinating seeds of Agriophyllum squarrosum (L.) Moq and Setaria viridis (L.) Beauv. Fresenius Environ. Bull. 30, 4730-4741 (2021).

13. Sheokand, S., Kumari, A. \& Sawhney, V. Effect of nitric oxide and putrescine on antioxidative responses under $\mathrm{NaCl}$ stress in chickpea plants. Physiol. Mol. Biol. Plants 14, 355-362 (2008).

14. Thakur, S. \& Kapila, S. Seasonal changes in antioxidant enzymes, polyphenol oxidase enzyme, flavonoids and phenolic content in three leafy liverworts. Lindbergia 5, 39-44 (2018).

15. An, M. J. et al. Effects of modifiers on the growth, photosynthesis, and antioxidant enzymes of cotton under cadmium toxicity. J. Plant Growth Regul. 10, 1-10 (2019).

16. Zhang, Z. F. \& Xiao, B. Z. Comparative alternative splicing analysis of two contrasting rice cultivars under drought stress and association of differential splicing genes with drought response QTLs. Euphytica 214, 73-89 (2018).

17. Mafakheri, A. et al. Effect of drought stress on yield, proline and chlorophyll contents in three chickpea cultivars. Aust. J. Crop Sci. 4, 580-585 (2010).

18. Guadagno, C. R. et al. Dead or alive? Using membrane failure and chlorophyll fluorescence to predict mortality from drought. Plant Physiol. 175, 223-234 (2017).

19. Li, G. L. et al. Response of chlorophyll fluorescence parameters to drought stress in sugar beet seedlings. Russ. J. Plant Physiol. 60, 337-342 (2013).

20. Guo, P. G. et al. QTLs for chlorophyll and chlorophyll fluorescence parameters in barley under post-flowering drought. Euphytica 163, 203-214 (2008).

21. Matos, M. C. et al. Drought effect on photosynthetic activity, osmolyte accumulation and membrane integrity of two Cicer arietinum genotypes. Photosynthetica 48, 303-312 (2010).

22. Xiao, X. W., Xu, X. \& Yan, F. Adaptive responses to progressive drought stress in two populous cathayana populations. Silva Fennica 42, 705-719 (2008).

23. Javadi, T. et al. Mitigating the adverse effects of drought stress on the morpho-physiological traits and anti-oxidative enzyme activities of Prunu savium through $\beta$-amino butyric acid drenching. Sci. Hortic. 218, 156-163 (2017).

24. Liu, C. et al. Responses of the antioxidant defense system to drought stress in the leaves of Fargesia denudata seedlings, the staple food of the giant panda. Russ. J. Plant Physiol. 61, 374-383 (2014).

25. Masoumi, H. et al. Effects of different levels of water deficit stresses on the morphologic properties of root, antioxidants activity and the seed yield in five cultivars of soybean (Glycine max L). J. Appl. Polym. Sci. 125, 804-811 (2014).

26. Alhoshan, M. et al. Effect of soil drought on biomass production, physiological attributes and antioxidant enzymes activities of potato cultivars. Russ. J. Plant Physiol. 66, 265-277 (2018)

27. Li, J. Y. et al. Historical grassland desertification changes in the Horqin Sandy Land, Northern China (1985-2013). Sci. Rep. 7, $1-12(2017)$.

28. Zhang, J. et al. Community succession along a chronosequence of vegetation restoration on sand dunes in Horqin Sandy Land. J. Arid Environ. 62, 555-566 (2005).

29. Huang, G. et al. Fine root dynamics and longevity of Artemisia halodendron reflect plant growth strategy in two contrasting habitats. J. Arid Environ. 79, 1-7 (2012).

30. Zhao, H. L. et al. Effects of desertification on soil and crop growth properties in Horqin sandy cropland of Inner Mongolia, north China. Soil Tillage Res. 87, 175-185 (2006).

31. Li, J. et al. Growth and physiological responses of Agriophyllum squarrosum to sand burial stress. J. Arid Land 7, 94-100 (2015).

32. Qu, H. et al. Effects of sand burial stress on maize (Zea mays L.) growth and physiological responses. Aust. J. Crop Sci. 6, 869-876 (2012).

33. Liu, H. et al. OsNOA1/RIF1 is a functional homolog of AtNOA1/RIF1: Implication for a highly conserved plant cGTPase essential for chloroplast function. New Phytol. 187, 83-105 (2010).

34. Krause, G. H. \& Weis, E. Chlorophyll fluorescence as a tool in plant physiology. Photosynth. Res. 5, 139-157 (1984).

35. Foad, M. \& Ismail, A. M. Responses of photosynthesis, chlorophyll fluorescence and ros-scavenging systems to salt stress during seedling and reproductive stages in rice. Ann. Bot. 6, 1161-1173 (2007).

36. Flexas, J. et al. Decreased rubisco activity during water stress is not induced by decreased relative water content but related to conditions of low stomatal conductance and chloroplast $\mathrm{CO}_{2}$ concentration. New Phytol. 172, 73-82 (2010).

37. An, Y. Y. \& Liang, Z. S. Drought tolerance of Periploca sepium during seed germination: Antioxidant defense and compatible solutes accumulation. Acta Physiol. Plant. 35, 959-967 (2013).

38. Giannopolitis, C. N. \& Ries, S. K. Superoxide dismutases: I. Occurrence in higher plants. Plant Physiol. 59, 309-314 (1977).

39. Heath, R. L. \& Packer, L. Photoperoxidation in isolated chloroplasts: I. Kinetics and stoichiometry of fatty acid peroxidation. Arch. Biochem. Biophys 125, 189-198 (1968).

40. Baker, N. R. Chlorophyll fluorescence: A probe of photosynthesis in vivo. Annu. Rev. Plant Biol. 59, 89-113 (2008).

41. Naumann, J. C., Young, D. R. \& Anderson, J. E. Linking leaf chlorophyll fluorescence properties to physiological responses for detection of salt and drought stress in coastal plant species. Physiol. Plant. 131, 422-433 (2010).

42. Vereb, G. et al. Dynamic, yet structured: The cell membrane three decades after the Singer-Nicolson model. Proc. Natl. Acad. Sci. 100, 8053-8058 (2003).

43. Soltys-Kalina, D. et al. The effect of drought stress on the leaf relative water content and tuber yield of a half-sib family of 'Katahdin'derived potato cultivars. Breed. Sci. 66, 328-331 (2016).

44. Zhao, H. L. et al. Effects of wind and wind-sand flow on membrane permeability and membrane protection system of corn seedling leaf. Chin. J. Eco-Agric. 22, 1440-1445 (2014) ((in Chinese)).

45. Luo, Y. Q. et al. Effects of foliage litter of a pioneer shrub (Artemisia halodendron) on germination from the soil seedbank in a semi-arid sandy grassland in China. J. Plant Res. 130, 1-9 (2017).

46. Mazorra, L. M. et al. Influence of brassinosteroids on antioxidant enzymes activity in tomato under different temperatures. Biol. Plant. 45, 593-596 (2002). 
47. Zhou, R. L. et al. The relationship between growth of Caraganas stenophylla and the activities and isoforms of protective enzymes under different depths of sand burial. Acta Ecol. Sin. 35, 3014-3022 (2015).

48. Chen, J. L. et al. Effects of precipitation on growth and physiology of three psammophytes in the Horqin Sandy Land, China. J. Desert Res. 39, 163-173 (2019) ((in Chinese)).

49. Bowler, C. Superoxide dismutase and stress tolerance. Annu. Rev. Plant Physiol. Plant Mol. Biol. 43, 83-116 (1992).

\section{Acknowledgements}

This study was financially supported by the National Basic Resources Investigation Program of China (2017FY100200), the National Natural Science Foundation of China (Grants 31500369, 41371053, and 31971466) and Research Fund of Mianyang Normal University (QD2021A02).

\section{Author contributions}

Conceptualization, X.Z. and Yu.L.; Investigation, J.C. and Yo.L.; Writing-original draft preparation, J.C.; Software, Yo.L and Y.Z.; Writing-review and editing, M.L. and Ya.L.; Data curation, J.C. and Y.Z.; X.Z identified modern vegetation information. All authors reviewed the manuscript.

\section{Competing interests}

The authors declare no competing interests.

\section{Additional information}

Correspondence and requests for materials should be addressed to J.C.

Reprints and permissions information is available at www.nature.com/reprints.

Publisher's note Springer Nature remains neutral with regard to jurisdictional claims in published maps and institutional affiliations.

(c) Open Access This article is licensed under a Creative Commons Attribution 4.0 International License, which permits use, sharing, adaptation, distribution and reproduction in any medium or format, as long as you give appropriate credit to the original author(s) and the source, provide a link to the Creative Commons licence, and indicate if changes were made. The images or other third party material in this article are included in the article's Creative Commons licence, unless indicated otherwise in a credit line to the material. If material is not included in the article's Creative Commons licence and your intended use is not permitted by statutory regulation or exceeds the permitted use, you will need to obtain permission directly from the copyright holder. To view a copy of this licence, visit http://creativecommons.org/licenses/by/4.0/.

(C) The Author(s) 2021 